\title{
Dynamics of $\mathrm{Mn}^{3+}$ in off-stoichiometric $\mathrm{LiMn}_{1.5} \mathrm{Ni}_{0.5} \mathrm{O}_{4}$
}

\author{
F.M. Vitucci ${ }^{\mathrm{a}, \mathrm{b}}$, O. Palumbo ${ }^{\mathrm{a}, \mathrm{c}}$, A. Paolone $^{\mathrm{a}, \mathrm{c}, *}$, R. Cantelli $^{\mathrm{a}, \mathrm{d}}, \mathrm{S}$. Brutti ${ }^{\mathrm{e}}, \mathrm{S}$. Panero ${ }^{\mathrm{a}, \mathrm{f}}$ \\ ${ }^{a}$ Research Center Hydro-Eco, Sapienza University of Rome, Via A. Scarpa 14, 00161 Roma, Italy \\ ${ }^{\mathrm{b}}$ Department SBAI, Sapienza University of Rome, Via A. Scarpa 14, 00161 Roma, Italy

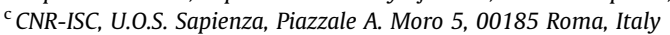 \\ d Physics Department, Sapienza University of Rome, Piazzale A. Moro 5, 00185 Roma, Italy \\ ${ }^{\text {e } C h e m i s t r y ~ D e p a r t m e n t, ~ U n i v e r s i t y ~ o f ~ B a s i l i c a t a, ~ P i a z z a l e ~ d e l l ' A t e n e o ~ L u c a n o, ~} 85100$ Potenza, Italy \\ ${ }^{\mathrm{f}}$ Chemistry Department, Sapienza University of Rome, Piazzale A. Moro 5, 00185 Roma, Italy
}

\section{A R T I C L E I N F O}

\section{Article history:}

Received 4 December 2013

Received in revised form 20 March 2014

Accepted 21 March 2014

Available online 31 March 2014

Keywords:

Electrode materials

Elasticity

Thermal analysis

\begin{abstract}
A B S T R A C T
$\mathrm{LiMn}_{1.5} \mathrm{Ni}_{0.5} \mathrm{O}_{4}$ is investigated by anelastic spectroscopy in order to study the manganese dynamics. An intense thermally activated peak, detectable only in off-stoichiometric samples, is attributed to a polaronic conduction due to an electron transfer from $\mathrm{Mn}^{3+}$ to a $\mathrm{Mn}^{4+}$, and the consequent motion of the Jahn-Teller lattice distortion. In order to have a good fit of the experimental data, the Jonscher model for ionic dynamics is adopted for the first time in this spinel structure. A value of about $0.3 \mathrm{eV}$ for the energy barrier of the polaronic conduction is obtained.
\end{abstract}

(c) 2014 Elsevier B.V. All rights reserved.

\section{Introduction}

Spinels derived from $\mathrm{LiMn}_{2} \mathrm{O}_{4}$ have been largely used as cathodes for lithium batteries because of their good stability, low cost and respect of environment [1-3]. However, on cycling $\mathrm{LiMn}_{2} \mathrm{O}_{4}$ presents a phase change [4] leading to rapid loss of capacity [5]. To overcome this loss, a family of $3: 1$ cation-ordered spinels, $\mathrm{LiMn}_{2-x} \mathrm{M}_{x} \mathrm{O}_{4}$ with $x=0.5 ; \mathrm{M}=\mathrm{Mg}, \mathrm{Cr}, \mathrm{Mn}, \mathrm{Fe}, \mathrm{Co}, \mathrm{Ni}, \mathrm{Zn}$, has been developed [6-8]. Among substituted spinels, $\mathrm{LiMn}_{1.5} \mathrm{Ni}_{0.5} \mathrm{O}_{4}$ has been largely investigated because it is cobalt free and is able to reversibly cycle lithium in electrochemical cells for hundreds of times at high current rates [9]. The high charge/discharge potential at $\sim 5 \mathrm{~V}[10]$ is caused by $\mathrm{Ni}^{2+}$ to $\mathrm{Ni}^{4+}$ oxidation, which completely replaces the oxidation process of the $\mathrm{Mn}^{4+} / \mathrm{Mn}^{3+}$ pair observed at ca. $4.0 \mathrm{~V}[11,12]$.

The structure and the performances of $\mathrm{LiMn}_{1.5} \mathrm{Ni}_{0.5} \mathrm{O}_{4}$ strongly depend on the preparation method [10-15]: high temperature synthesis produces a face-centered cubic spinel, $\mathrm{Fd}-3 \mathrm{~m}$ space group, cF56 lattice, whereas samples annealed below $700{ }^{\circ} \mathrm{C}$ present an ordered spinel with a $\mathrm{P}_{3} 2$ space group $[13,14]$. Moreover, samples synthesized above $650^{\circ} \mathrm{C}$ lose oxygen and separate in a spinel phase with a smaller $\mathrm{Ni}$ content and in a $\mathrm{Li}_{x} \mathrm{Ni}_{1-x} \mathrm{O}_{1-x / 2}$ phase

* Corresponding author at: CNR-ISC, U.O.S. Sapienza, Piazzale A. Moro 5, 00185 Roma, Italy. Tel.: +39 (0) 649914400; fax: +39 (0) 64957697.

E-mail address: annalisa.paolone@roma1.infn.it (A. Paolone).
(Fm-3m space group, cF8 lattice). This leads to the occurrence of some $\mathrm{Mn}^{3+}$ and causes the development of a $4-\mathrm{V}$ plateau and a decrease in 5-V capacity [11]. Despite these drawbacks, in many cases syntheses at higher temperatures are preferred, because the growth of the spinel phase is extremely slow when conducted below $650{ }^{\circ} \mathrm{C}$. A detailed investigation [9] on the effects of the synthesis conditions on the materials structure, morphology and performances showed that samples produced by wet chemistry, starting from acetate precursors and annealed at $800^{\circ} \mathrm{C}$, were the best performing.

In the present work we investigated the presence and the dynamics of defects in $\mathrm{LiMn}_{1.5} \mathrm{Ni}_{0.5} \mathrm{O}_{4}$ by means of anelastic spectroscopy (AS), in particular of the $\mathrm{Mn}^{3+}$ ions which, even though undesired, in most cases are present in the spinels. It is shown that $\mathrm{Mn}^{3+}$ Jahn-Teller ions can transfer an electron to $\mathrm{Mn}^{4+}$ sites; the consequent motion of the electron and its associated lattice distortion gives rise to a polaron dynamics revealed by AS.

\section{Experimental}

The $\mathrm{LiNi}_{0.5} \mathrm{Mn}_{1.5} \mathrm{O}_{4}$ powders were synthesized by wet chemistry method using metallic acetate precursors [9]. Inductively Coupled Plasma Atomic Absorption (ICP-AA) has been used to confirm the overall stoichiometry of the samples. The obtained materials were characterized by means of structural (X-ray Diffraction) and electrochemical (Galvanostatic Cycling) techniques. The XRD experiments were carried out using Philips X'Pert Pro diffractometer equipped with a fast X'celerator detector and a $\mathrm{Cu} \mathrm{K}$ radiation source. The Rietveld refinement of the recorded spectra was performed by the software Maud $[16,17]$. 
The consolidated samples necessary to perform anelastic spectroscopy measurements were obtained by mixing the spinel with $\mathrm{KBr}$ powder and pressing the mixture in a rectangular die, with dimensions of $40 \times 6 \mathrm{~mm}^{2}$, following a well established procedure [18-21].

The bars used for anelastic spectroscopy measurements were suspended on thin wires located at the nodal lines of flexural vibration modes and were excited to the corresponding mechanical resonances by means of an alternate electric field.

The sample vibration produces an alternate stress which interacts with the local lattice distortions introduced by the mobile entities and perturbs their site energies in such a way that the sites that are energetically favored in the first half period become unfavored in the second half. The system then attempts to reach an equilibrium redistribution among the perturbed levels. At the temperature at which the relaxation rate $\tau^{-1}$ of the mobile atoms is equal to the angular vibration frequency $\omega$, i.e. at the Debye relaxation condition $\omega \tau=1$, the stimulated atomic migration follows, by thermal activation, the sample vibration and the coefficient of elastic energy dissipation $Q^{-1}$ reaches its maximum value.

The energy dissipation (or reciprocal of the mechanical quality factor $Q$ ) is measured from the width of the resonance peak. The dynamic Young's modulus $E^{\prime}$ is obtained together with $Q^{-1}$ from the angular vibration frequency $(\omega): E^{\prime}=\omega^{2} \rho / z$, where $\rho$ is the mass density and $z$ a numerical factor depending on the sample geometry [22].

As described above, in case a species can move in the sample with a relaxation rate $\tau^{-1}$ by means of thermal activation, the elastic energy coefficient presents a maximum when the Debye relaxation condition $\omega \tau=1$ is satisfied. For a single relaxation time, $\tau, Q^{-1}$ is given by:

$Q^{-1}=\Delta \frac{1}{(\omega \tau)^{-\alpha}+(\omega \tau)^{\alpha}}=g v_{0}\left(\lambda_{1}-\lambda_{2}\right)^{2} \frac{c n_{1} n_{2}}{k T} E^{\prime} \frac{1}{(\omega \tau)^{-\alpha}+(\omega \tau)^{\alpha}}$

where $c$ is the molar concentration of the jumping atoms and $n_{1}$ and $n_{2}$ their equilibrium fractions in sites 1 and $2 ; \lambda_{1}$ and $\lambda_{2}$ are the elastic dipoles [22] of the defects in their two configurations; $g$ is a factor of the order of $1 / 2$ depending on the geometry of the jump and the type of sample vibration, $v_{0}$ the unit cell volume, $\alpha$ a parameter equal to 1 for a single Debye process, and $k$ the Boltzmann constant. For classical processes $\tau=\tau_{0} e^{W / k T}$, where $W$ is the activation energy. In many cases, the experimental peaks are broader than the Debye one and they can be described by means of a Fuoss-Kirkwood model, in which the parameter $\alpha$ in Eq. (1) becomes smaller than 1.

In the usual model of defect relaxation between two equivalent sites, the relaxation intensity $(\Delta)$ in Eq. (1) decreases with increasing $T$, leading to a higher intensity for the peaks measured at lower frequencies. However, experimentally, many peaks are more intense when measured at higher frequencies. In such cases, one has to consider the process of hopping between two non equivalent sites, 1 and 2 , with energy separation $\Delta E$ : the relaxation intensity, which is proportional to the product of the respective populations, becomes [23]:

$\frac{c n_{1} n_{2}}{T} \propto \frac{c}{T} \sec h^{2}\left(\frac{\Delta E}{2 k T}\right)$

and leads to higher intensity of peaks detected at higher $\omega$. Moreover, many materials present highly asymmetrical peaks, having a large low temperature tail, which cannot be described by use of a physically reasonable distribution of relaxation times [24]. In this cases a satisfactory analysis can be obtained [25] replacing the $\omega \tau$ Debye-like formula by the Jonscher empirical expression of the dielectric response theory [26]:

$Q^{-1} \propto \Delta \frac{1}{\left(\omega / \omega^{\prime}\right)^{-m}+\left(\omega / \omega^{\prime}\right)^{1-n}}$

In this model, the temperature dependence of the relaxation intensity $(\Delta)$ is retained [22]; the factors $n$ and $m$ are lower than 1; the parameter $\omega^{\prime}$ is expected to be of the same order of magnitude of $\tau^{-1}$. The parameter $\omega^{\prime}$ is assumed to be $\omega^{\prime}=\omega_{0}^{\prime} e^{-W / k T}$ and is used to measure the activation energy, $W$, in the usual way [25].

Thermogravimetry (TGA) measurements were performed concomitantly with mass spectrometry (MS), using a Setaram Setsys Evolution 1200 TGA System, equipped with a Pfeiffer Prisma analyzer QMS 200. The TGA curves were measured during heating with a rate of $10 \mathrm{~K} / \mathrm{min}$ in a vacuum of the order of $10^{-4} \mathrm{mbar}$. The cooling was carried out at the same pressure and with the same temperature rate.

\section{Results and discussion}

Fig. 1 shows the X-ray diffraction analysis of the as prepared sample (sample S1). The pristine material is constituted by the cF56 lattice (crystallite size $194 \mathrm{~nm}$, consistent with the stoichiometry $\mathrm{Li}_{0.98} \mathrm{Ni}_{0.46} \mathrm{Mn}_{1.54} \mathrm{O}_{4}$ ) with some traces of the cF8 phase (2.7 vol.\%, crystallite size $49 \mathrm{~nm}$, consistent with the stoichiometry $\left.\mathrm{Li}_{0.1} \mathrm{Ni}_{0.9} \mathrm{O}_{0.95}\right)$. The resulting mean $\mathrm{Mn}$ oxidation state of the spinel phase is 3.96 to be compared with the 3.98 estimate from CG data

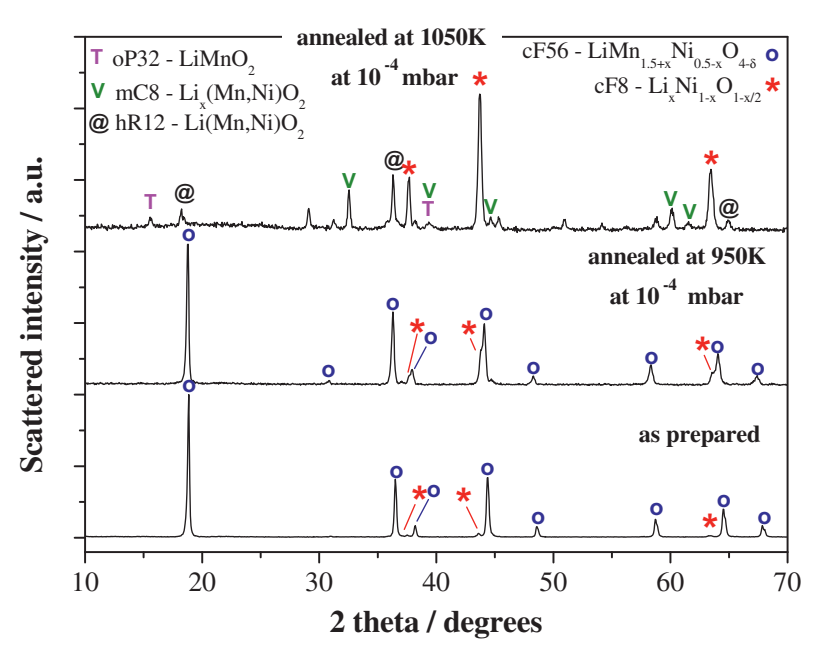

Fig. 1. X-ray diffraction patterns of samples S1 (as prepared), S3 (annealed at $10^{-4}$ mbar at $950 \mathrm{~K}$ ) and S4 (annealed at $10^{-4} \mathrm{mbar}$ at $1050 \mathrm{~K}$ ).

(results not shown), indicating a content of $\mathrm{Mn}^{3+}<4 \%$ of the total manganese ions.

Fig. 2 shows the anelastic spectrum of the as-prepared S1 sample, measured in the temperature range between $290 \mathrm{~K}$ and $4 \mathrm{~K}$ and the featureless dissipation of a pure $\mathrm{KBr}$ sample, to be considered as a background. The spectrum of the as-received sample S1 displays a broad dissipation peak centered around $100 \mathrm{~K}$ and a smaller bump around $230 \mathrm{~K}$. The spectrum was measured at different frequencies (results not shown) but the broadness of these features made difficult to ascertain if they were due to thermally activated processes or not. In this compound, non thermally activated processes could be induced by the occurrence of magnetic transitions in the $\mathrm{LiNi}_{0.5} \mathrm{Mn}_{1.5} \mathrm{O}_{4}$-like spinel $[27,28]$ and in the spurious $\mathrm{Li}_{x} \mathrm{Ni}_{1-x} \mathrm{O}_{1-x / 2}$ phase $[29,30]$. On the contrary, the dynamics of $\mathrm{Mn}^{3+}$ would give rise to a thermally activated relaxation process, as already reported for the $\mathrm{LiMn}_{2} \mathrm{O}_{4}$ parent compound [31].

To quantitatively investigate the dynamics of $\mathrm{Mn}^{3+}$ ions, we increased their concentration by means of thermal treatments. It is known [32,33] that an oxygen off-stoichiometry is balanced by a change in the $\mathrm{Mn}$ ions valence. Therefore, we prepared a $\mathrm{Mn}^{3+}$

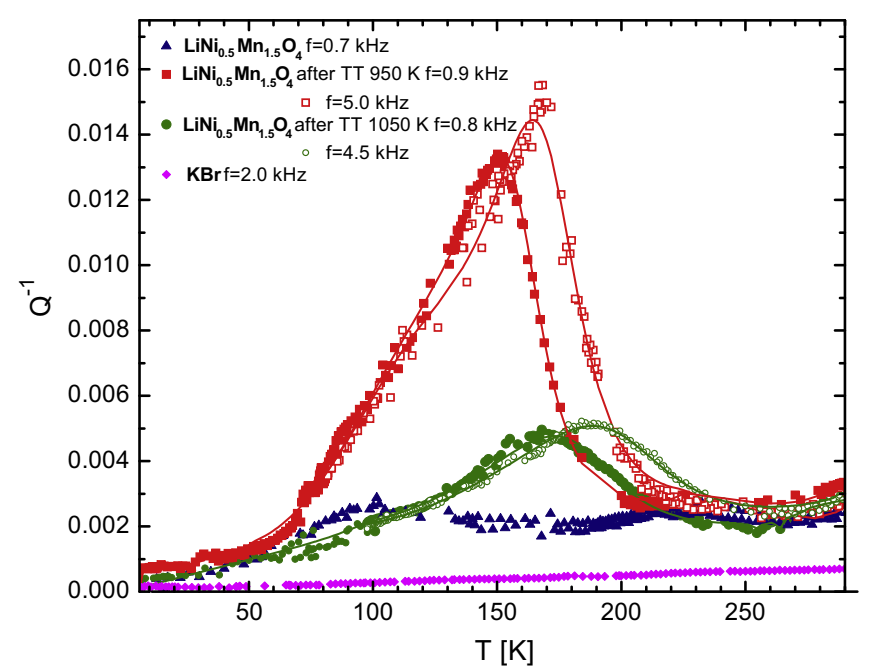

Fig. 2. Elastic energy loss of sample $\mathrm{S} 3\left(\mathrm{LiNi}_{0.5} \mathrm{Mn}_{1.5} \mathrm{O}_{4}\right.$ heated in vacuum up to $950 \mathrm{~K})$ and $\mathrm{S} 4\left(\mathrm{LiNi}_{0.5} \mathrm{Mn}_{1.5} \mathrm{O}_{4}\right.$ heated in vacuum up to $\left.1050 \mathrm{~K}\right)$. Lines are best fit curves. As a reference also the spectra of sample $\mathrm{S} 1$ (starting $\mathrm{LiNi}_{0.5} \mathrm{Mn}_{1.5} \mathrm{O}_{4}$ spinel) and of $\mathrm{KBr}$ are shown. 
enriched samples by heating the starting spinel in vacuum $\left(10^{-4} \mathrm{mbar}\right)$ in a thermobalance. TGA data are reported in Fig. 3 and show that the mass starts to decrease at $850 \mathrm{~K}$; the loss is about $1.0 \%$ at $950 \mathrm{~K}$ and reaches a value of about $5.4 \%$ at $1050 \mathrm{~K}$, and it is entirely due to the release of oxygen, as indicated by the concomitant MS measure. The oxygen loss induces the presence of $15 \%(41 \%) \mathrm{Mn}^{3+}$ ions in the sample heated at $950 \mathrm{~K}(1050 \mathrm{~K})$.

XRD experiments performed on sample S3 (heated in vacuum at $950 \mathrm{~K}$ ) confirm that the pristine cF56 phase (the spinel $\mathrm{LiNi}_{0.5} \mathrm{Mn}_{1.5} \mathrm{O}_{4}$ phase) is the dominant phase and the amount of the contaminant cF8 lattice (i.e. $\mathrm{Li}_{x} \mathrm{Ni}_{1-x} \mathrm{O}_{1-x / 2}$ ) is only slightly increased ( 8.5 vol.\%) (see Fig. 1). The anelastic spectrum of sample $\mathrm{S} 3$ (with $15 \% \mathrm{Mn}^{3+}$ ) measured between 300 and $4 \mathrm{~K}$ displays a very intense thermally activated peak centered around $150 \mathrm{~K}$ with a shoulder on the low temperature side (see Fig. 2). The peak is quite large and highly asymmetric and its shape closely resembles the one of the relaxation peak previously measured in $\mathrm{LiMn}_{2} \mathrm{O}_{4}$ [31].

An analysis of the peaks by means of the Fouss-Kirkwood model did not account for the width of the peaks and for their shape, as already observed in $\mathrm{LiMn}_{2} \mathrm{O}_{4}$ [31]. Therefore, we adopted the more complex Jonscher empirical formula of the universal dielectric response theory (Eq. (3)), considering also the temperature dependence of the relaxation intensity $(\Delta)$ for double potential well, with two non-equivalent sites (Eq. (2)):

$Q_{J}^{-1}=\frac{C}{T} \operatorname{sech}^{2}\left(\frac{\Delta E}{2 k T}\right) \frac{1}{\left(\omega / \omega_{0}^{\prime} e^{-W / k T}\right)^{-m}+\left(\omega / \omega_{0}^{\prime} e^{-W / k T}\right)^{1-n}}$

Moreover, we added a gaussian contribution to the elastic dissipation, $Q_{G}^{-1}$, to account for possible non thermally activated contributions due to the occurrence of magnetic phase transitions in the $\mathrm{LiNi}_{0.5} \mathrm{Mn}_{1.5} \mathrm{O}_{4}$ spinel and the spurious $\mathrm{Li}_{x} \mathrm{Ni}_{1-x} \mathrm{O}_{1-x / 2}$ phase [2730]:

$Q_{G}^{-1}=\frac{A}{l \sqrt{\pi / 2}} e^{-2\left(\left(T-T_{C}\right) / l\right)^{2}}$

The best fit curves are plotted as lines in Fig. 2 and the relative parameters are reported in Table 1 . The activation energy, $W$, and the energy separation, $\Delta E$, obtained from the Jonscher model for the mobile species are $W=0.301 \mathrm{eV}$ and $\Delta E=0.053 \mathrm{eV}$. As discussed in Section 2, the parameter $\omega^{\prime}$ is assumed to be $\omega^{\prime}=\omega^{\prime} e^{-W / k T}$; even though it does not provide information on $\tau$, it is used to measure the activation energy $W$.

The peak at $150 \mathrm{~K}$ is likely originated by the presence of $\mathrm{Mn}^{3+}$ ions. Indeed, also $\mathrm{O}$ vacancies are present in the samples, but they give rise to symmetric peaks [22]. The need of fitting the anelastic

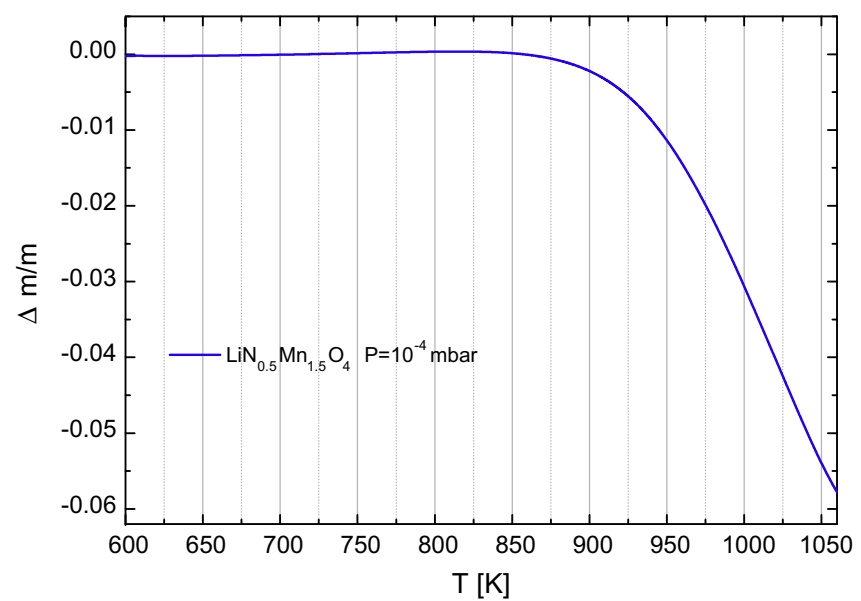

Fig. 3. TG curve of the starting $\mathrm{LiNi}_{0.5} \mathrm{Mn}_{1.5} \mathrm{O}_{4}$ powders on heating up to $1050 \mathrm{~K}$.
Table 1

Parameters of the best fit curves.

\begin{tabular}{lcc}
\hline & $\mathrm{S} 3$ & $\mathrm{~S} 4$ \\
\hline$\Delta E(\mathrm{eV})$ & 0.053 & 0.035 \\
$\tau_{0}(\mathrm{~s})$ & $6.1 \mathrm{E}-14$ & $5.9 \mathrm{E}-13$ \\
$W(\mathrm{eV})$ & 0.301 & 0.320 \\
$m$ & 0.14 & 0.10 \\
$n$ & 0.01 & 0.18 \\
$C(\mathrm{~K})$ & 31 & 2.1 \\
$A(\mathrm{~K})$ & 0.34 & 0.00231 \\
$l(\mathrm{~K})$ & 55 & 70 \\
$T_{\mathrm{c}}(\mathrm{K})$ & 117 & 127 \\
\hline
\end{tabular}

peak with a model based on ionic conduction [25] further corroborate the hypothesis of the motion of manganese ions. Moreover, the ionic dynamics was already reported for spinels containing mixed valence ions, like $\mathrm{LiMn}_{2} \mathrm{O}_{4}, \mathrm{Fe}_{3} \mathrm{O}_{4}$ and $\mathrm{FeMnO}_{4}$ [31-34]. It is important to note that the presence of $\mathrm{Mn}^{3+}$ would induce a transfer of an electron from a $\mathrm{Mn}^{3+}$ to $\mathrm{Mn}^{4+}$ ion. Anelastic spectroscopy would not detect a transfer of charge, if it was not accompanied by a lattice distortion [22]. However, $\mathrm{Mn}^{3+}$ is a Jahn-Teller ion, which implies that the lattice is distorted around it; during the charge transfer to $\mathrm{Mn}^{4+}$, the motion of the electron is associated with the lattice distortion and gives rise to a polaron dynamics revealed by AS. One can note that the present analysis provide for the first time the activation energy for the movement of $\mathrm{Mn}^{3+}$ ions in $\mathrm{LiNi}_{0.5} \mathrm{Mn}_{1.5} \mathrm{O}_{4}$ and a good fitting model which is able to reproduce the experimental data quite well.

Previous literature about the movement of polarons in the parent compound $\mathrm{LiMn}_{2} \mathrm{O}_{4}$ reported great discrepancies in the values of the activation energy: Sugiyama et al. [34] reported a value of about $0.14 \mathrm{eV}$ from the Arrhenius plot, while, later on, Paolone et al. [31] obtained a value of $0.062 \mathrm{eV}$ by means of a Fouss-Kirkwood model. This difference was attributed to a different stoichiometry of the samples, but it is worth noting that the models used in those papers are not able to well reproduce the experimental data. In the present work the Jonscher formula is finally able to give a good fitting of the experimental points.

To investigate the effects of an additional increase of number of the $\mathrm{Mn}^{3+}$ ions, we measured the anelastic spectrum of a sample (sample S4) heated in vacuum up to $1050 \mathrm{~K}$, inducing about $41 \%$ of $\mathrm{Mn}^{3+}$. S4 displays a thermally activated relaxation peak (see Fig. 2), which is less intense and shifted to higher temperature with respect to the one observed for the sample S3. This peak was analyzed by means of the same model used for sample S3 and the best fit curves obtained from the analysis are plotted as lines in Fig. 2 and the relative parameters are reported in Table 1. In S4 the non thermally activated component is practically negligible. The activation energy, $W$, and the energy separation, $\Delta E$, obtained from the Jonscher model are in good agreement with those ones obtained for sample S3, but the values of $\omega^{\prime}$ in S4 is higher than in S3 by an order of magnitude $\left(6 \times 10^{-13} \mathrm{~s}\right.$ vs. $\left.6 \times 10^{-14} \mathrm{~s}\right)$, and therefore also the $\tau_{0}$ would differ by an order of magnitude in the two samples. These differences for the relaxation processes observed in the two samples S3 and S4, i.e. the absence of non thermally activated contribution and the different values of the parameters, can be ascribed to the a dramatic change induced in the lattice by the thermal treatment up to $1050 \mathrm{~K}$. In particular one can connect the absence of the peak around $117 \mathrm{~K}$ to the disappearance of the spinel phase, which undergoes a magnetic phase transition around such temperature. Indeed X-ray Diffraction on the material heated in vacuum up to $1050 \mathrm{~K}$ (Fig. 1) showed that the pristine cF56 phase completely disappeared and the cF8 phase becomes the dominant component of the material. The possible other components tentatively identified in the XRD pattern of the 
material heated at $1050 \mathrm{~K}$ were mixed oxides $\left(\mathrm{Li}_{x}(\mathrm{Mn}, \mathrm{Ni}) \mathrm{O}_{2}(\mathrm{oP} 32\right.$, hR12 or mC8 lattices) [35-38], where large part of Mn ions are 3+. Indeed the relaxation process observed in sample $S 4$ could be due to the dynamics of $\mathrm{Mn}^{3+}$ ions relaxing in a lattice strongly modified by the oxygen loss, but the possibility that the peak could be ascribed to a different relaxing unit cannot be ruled out.

\section{Conclusions}

In $\mathrm{LiNi}_{0.5} \mathrm{Mn}_{1.5} \mathrm{O}_{4}$ samples, heat-induced oxygen deficiency increases the presence of $\mathrm{Mn}^{3+}$. In the anelastic spectra of these samples, the dynamics of the manganese ions is detected as a thermally activated process, which would correspond to a transfer of an electron from a $\mathrm{Mn}^{3+}$ to $\mathrm{Mn}^{4+}$ ion. Since $\mathrm{Mn}^{3+}$ is a Jahn-Teller ion, the lattice is distorted around it; therefore during the hopping of $\mathrm{Mn}^{3+}$ a transfer of the distortion of the lattice from a lattice site to another is expected. In other words, the dynamics is of polaronic origin. It is possible to model such polaronic dynamics by means of the Jonscher model which provides an activation energy of $0.3 \mathrm{eV}$.

\section{Acknowledgments}

One of the authors (F.M.V.) thanks the European Community for a grant within the Seventh Framework Program APPLES (Advanced, High Performance, Polymer Lithium Batteries for Electrochemical Storage) Project (contract number 265644).

\section{References}

[1] H. Kawai, M. Nagata, H.R. Tukamoto, A. West, J. Power Sources 81 (1999) 67-

[2] T. Ohzuku, K. Ariyoshi, S. Yamamoto, Y. Makimura, Chem. Lett. 30 (2001) 1270.

[3] L. Hernán, J. Morales, L. Sánchez, E. Rodríguez Castellón, M.A. Aranda, J. Mater. Chem. 12 (2002) 734-741.

[4] Q. Liu, D. Mao, C. Chang, F. Huang, J. Power Sources 173 (2007) 538.

[5] T. Matsushima, J. Power Sources 189 (2009) 847.

[6] S. Mandal, R.M. Rojas, J.M. Amarilla, P. Calle, N.V. Kosova, V.F. Anufrienko, J.M. Rojo, Chem. Mater. 14 (2002) 1598.

[7] H. Shigemura, M. Tabuchi, H. Kobayashi, H. Sakaebe, A. Hirano, H. Kageyama, J. Mater. Chem. 12 (2002) 1882.
[8] R. Alcántara, M. Jaraba, P. Lavela, J.L. Tirado, Chem. Mater. 15 (2003) 1210.

[9] L.H. Chi, N.N. Dinh, S. Brutti, B. Scrosati, Electrochim. Acta 55 (2010) 5110.

[10] T. Ohzuku, S. Takeda, M. Iwanaga, J. Power Sources 81 (1999) 90.

[11] Q. Zhong, A. Bonakdarpour, M. Zhang, Y. Gao, J.R. Dahn, J. Electrochem. Soc. 144 (1997) 205.

[12] D. Aurbach, B. Markovsky, G. Salitra, E. Markevich, Y. Talyossef, M. Koltypin, L. Nazar, B. Ellis, D. Kovacheva, J. Power Sources 165 (2007) 491

[13] R. Alcantara, M. Jaraba, P. Lavela, J.L. Tirado, Electrochim. Acta 47 (2002) 1829

[14] K. Takahashi, M. Saitoh, M. Sano, M. Fujita, K. Kifune, J. Electrochem. Soc. 151 (2004) 173.

[15] Y. Idemoto, H. Narai, N. Koura, J. Power Sources 119-121 (2003) 125-129.

[16] M. Ferrari, L. Lutterotti, J. Appl. Phys. 76 (1994) 7246.

[17] H.-R. Wenk, S. Matthies, L. Lutterotti, Mater. Sci. Forum 157 (1994) 473.

[18] A. Paolone, R. Cantelli, R. Caciuffo, A. Arduini, Phys. Rev. B65 (2002) 214304.

[19] O. Palumbo, A. Paolone, R. Cantelli, C.M. Jensen, M. Sulic, J. Phys. Chem. B 110 (2006) 9105-9111.

[20] O. Palumbo, A. Paolone, R. Cantelli, C.M. Jensen, R. Ayabe, Mat. Sci. Eng. A 442 (2006) 75-78.

[21] R. Cantelli, O. Palumbo, A. Paolone, C.M. Jensen, M.T. Kuba, R. Ayabe, J. Alloys Comp. 446-447 (2007) 260-263.

[22] A.S. Nowick, B.S. Berry, Anelastic Relaxation in Crystalline Solids, Academic Press, New York, 1972.

[23] G. Cannelli, R. Cantelli, F. Cordero, F. Trequattrini, Phys. Rev. B 55 (1997) $14865-14871$.

[24] M. Barmatz, R. Farrow, in: J. de Klerk, B. McAvoy (Eds.), Proceedings of the Ultrasonic Symposium, Institute of Electrical and Electronics Engineers, New York, 1976, p. 62.

[25] D.P. Almond, A.R. West, Phys. Rev. Lett. 47 (1981) 431-435.

[26] A.K. Jonscher, Nature 256 (1975) 566.

[27] W. Branford, M.A. Green, Chem. Mater. 14 (2002) 1649-1656.

[28] C. Castellano, F. Cordero, R. Cantelli, M. Ferretti, J. Appl. Phys. 92 (2002) 7206 7209.

[29] A. Bajpai, A. Banerjee, Phys. Rev. B 55 (1997) 12439-12445.

[30] A. Bajpai, A. Banerjee, J. Phys.: Condens. Matter 13 (2001) 637.

[31] A. Paolone, R. Cantelli, G. Rousse, C. Masquelier, J. Phys.: Condens. Matter 15 (2007) 457-465

[32] A. Caballero, M. Cruz, L. Hernan, M. Melero, J. Morales, E. Rodrıguez Castellon, J. Electrochem. Soc. 152 (2005) A552-A559.

[33] J. Arrebola, A. Caballero, L. Hernan, J. Morales, J. Power Sources 180 (2008) $852-858$.

[34] J. Sugiyama, T. Tamura, H. Yamauchi, J. Phys.: Condens. Matter 7 (1995) 9755 9764.

[35] D.G. Kellerman, J.E. Medvedeva, V.S. Gorshkov, A.I. Kurbakov, V.G. Zubkov, A.P. Tyutyunnik, V.A. Trunov, Solid State Sci. 9 (2007) 196-204.

[36] A.R. Armstrong, P.G. Bruce, Nature 38 (1996) 499-500.

[37] J. Breger, N. Dupre, P.J. Chupas, P.L. Lee, T. Proffen, J.B. Parise, C.P. Grey, J. Am. Chem. Soc. 127 (2005) 7529-7537.

[38] Y. Arachi, H. Kobayashi, S. Emura, Y. Nakata, M. Tanaka, T. Asai, H. Sakaebe, K. Tatsumi, H. Kageyama, Solid State Ionics 176 (2005) 895-903. 